Radiologe 2022 $62: 197-199$

https://doi.org/10.1007/s00117-022-00978-9

Angenommen: 1. Februar 2022

(C) The Author(s), under exclusive licence to Springer Medizin Verlag GmbH, ein Teil von Springer Nature 2022

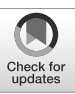

\section{Autor}
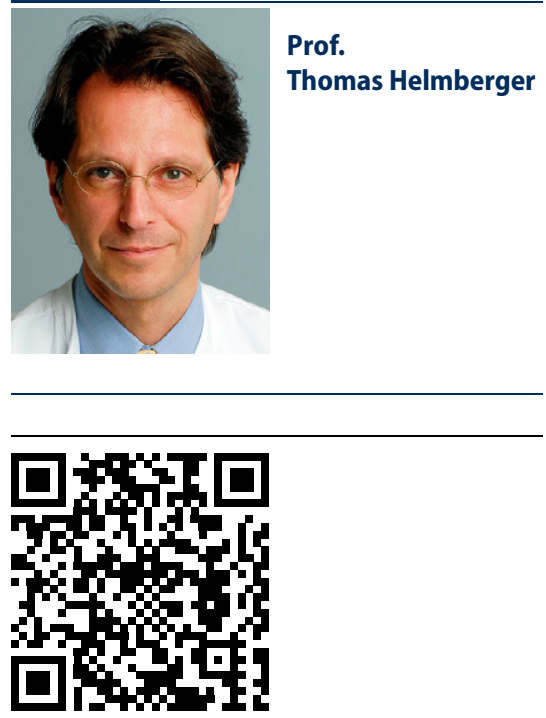

QR-Code scannen \& Beitrag online lesen

\title{
Leitlinie zum hepatozellulären Karzinom
}

Thomas Helmberger

Institut für Radiologie, Neuroradiologie und minimal-invasive Therapie, Klinikum Bogenhausen, München, Deutschland

Liebe Leserinnen und Leser,

das hepatozelluläre Karzinom (HCC) zählt mit einer Häufigkeit von etwa $6 \%$ bei Männern und $3 \%$ bei Frauen zu den häufigsten Karzinomen weltweit. Die Inzidenz ist insbesondere in Regionen wie Asien und Afrika, mit hohen Raten an Virushepatitiden $(B, C)$ und Toxinen wie Aflatoxinen besonders hoch. Dennoch ist in den letzten 10-15 Jahren gerade in den westlichen Ländern ein deutlicher Inzidenzanstieg für das HCC zu verzeichnen. So kam es in Deutschland in diesem Zeitraum ebenfalls zu einer deutlichen Zunahme von ca. 7/100.000 auf 11-12/100.000 Fällen/ Jahr [1].

Dies liegt im Wesentlichen an chronischen Hepatitis-C-Infektionen, Alkoholkonsum und der signifikanten Zunahme an nichtalkoholischen Fettlebererkrankungen (NAFLD) mit nichtalkoholischen Fettleberhepatitiden (NASH) mit fortgeschrittener Fibrose oder Zirrhose.

Nachdem die letzte nationale Leitlinie zum HCC (s. Der Radiologe 07/2014: Leitthema: Hepatozelluäres Karzinom) 2013 veröffentlicht wurde, machten die diagnostischen und therapeutischen Entwicklungen der letzten Jahre eine Neufassung dringend notwendig, die gleichzeitig um die biliären Karzinome erweitert wurde.

Zahlreiche Fachdisziplinen sind in die Diagnostik und Therapie der hepatobiliären Tumoren involviert, was die Erstellung einer Leitlinie zu einer sehr komplexen Aufgabe werden lässt. Unter dem Prozessmonitoring der AWMF (Arbeitsgemeinschaft der Wissenschaftlichen Medizinischen Fachgesellschaften e. V.) haben sich die Vertreter von 35 Fachgesellschaften dieser Herausforderung gestellt, den aktuellen Stand der Wissenschaft zur Behandlung von hepatobiliären Tumoren analysiert und eine umfassende Leitlinie mit zahlreichen Änderungen im Vergleich zur früheren und vielen anderen internationalen Leitlinien erstellt [2].

So wurde für die allgemeinen Therapieentscheidungen die Bedeutung des interdisziplinären Tumorboards deutlich betont, ebenso die der kontrastmittelverstärkten Magnetresonanztomographie (MRT) und der Biopsie in der primären Diagnose hepatobiliärer Tumoren.

Beim frühen HCC (Stadium A) haben sich bei der Lebertransplantation neben den Mailand-Kriterien auch erweiterten Selektionskriterien, wie z. B. die UCSF-Kriterien, nach einem entsprechenden Downstaging etabliert, was die Transplantationsmöglichkeiten deutlich erweitert. Neuere Resektionstechniken sowie die verschiedenen radiologischen minimal-invasiven Therapieverfahren werden im frühen Stadium sowohl als kurative Therapien als auch zum Downstaging und Bridging vor Transplantation angeboten. Beim intermediären HCC (Stadium B) gilt weiterhin die transarterielle Chemoembolisation als Methode der Wahl, wobei auch die Rolle der Radioembolisation unter Berücksichtigung der großen Studien der letzten Jahre differenziert miteinbezogen wird. Erstmals wurde in der aktuellen Leitlinie das Konzept der Stadienmigration in die Empfehlungen integriert, d.h. das Downstaging durch insbesondere minimal-invasive Therapien von einem höheren Tumorstadium (C) zu einem niedrigeren mit weiteren Therapieoptionen.

Im fortgeschrittenen Stadium C waren die bisherigen systemischen Therapieoptionen sehr beschränkt und im Ergebnis 
eher enttäuschend. Die aktuellen Ergebnisse zu neuen systemischen Therapien mit Antikörpern (PD-L1-AK, VEGF-AK) oder Tyrosinkinaseinhibitoren, sind als neue palliative Therapieoptionen in die Empfehlungen eingegangen, wie sie nun auch zuletzt in die Empfehlungen der Barcelona Clinic Liver Cancer (BCLC) aufgenommen wurden [3].

Darüber hinaus wurden bei zwar noch begrenzter Datenlage erstmals auch Empfehlungen zur Diagnostik und Therapie intrahepatischer biliärer Karzinom definiert.

Es ist gelungen, zahlreiche Mitglieder aus der Leitliniengruppe als Autorinnen/ Autoren für dieses Themenheft zu gewinnen, die die Inhalte der Leitlinie für die verschiedenen Themenbereiche quasi aus erster Hand für Sie aufbereitet haben dafür sei den Autorinnen und Autoren an dieser Stelle herzlichst gedankt.

Viel Spaß beim Lesen mit hoffentlich vielen Anregungen und Unterstützung für Ihre tägliche Arbeit,

Ihr

Thomas Helmberger

\section{Korrespondenzadresse}

\section{Prof. Thomas Helmberger}

Institut für Radiologie, Neuroradiologie und minimal-invasive Therapie, Klinikum Bogenhausen

Englschalkinger Str. 77, 81925 München, Deutschland

thomas.helmberger@muenchen-klinik.de

Interessenkonflikt. T. Helmberger gibt an, dass kein Interessenkonflikt besteht.

\section{Literatur}

1. http://krebsdaten.de.Zugegriffen: 14. Feb. 2022

2. https://www.awmf.org/uploads/tx_szleitlinien/ 032-0530LI_S3_HCC_biliäre_Karzinome_202106.pdf. Zugegriffen: 14 . Feb. 2022

3. Reig M et al (2021) BCLC strategy for prognosis prediction and treatment recommendation: the 2022 update. J Hepatol. https://doi.org/10.1016/j. jhep.2021.11.018

\section{Prototyp kombiniert klassische Röntgen-CT und Dunkelfeld-Röntgenverfahren}

Ein Forschungsteam der Technischen Universität München (TUM) hat erstmalig eine neue Röntgenmethode, das Dunkelfeld-Röntgen, in einen für den Patienteneinsatz ausgelegten Computertomographen integriert. Dunkelfeld-Röntgen liefert zusätzliche Informationen zu konventionellen Röntgenaufnahmen.

Die Computertomographie (CT) ist eine der wichtigsten Methoden in Kliniken für präzise und schnelle Diagnosen. Bisher werden dafür konventionelle Röntgenaufnahmen verwendet, um diese dann in ein dreidimensionales CT-Bild umzurechnen.

Eine neue Röntgentechnologie, das Dunkelfeld-Röntgen, kann zusätzliche Informationen liefern und feine Gewebestrukturen, insbesondere der Lunge, deutlich detaillierter als bisher abbilden. Bisher gab es jedoch aufgrund technischer Herausforderungen keine Möglichkeit, Patientinnen und Patienten mit der neuen Röntgentechnologie in einem klinischen CT-Gerät zu untersuchen.

Ein Forschungsteam um Franz Pfeiffer, Professor für biomedizinische Physik und Direktor des Munich Institute of Biomedical Engineering der TUM, hat nun ein CT-Gerät so weiterentwickelt, dass es beide Röntgentechnologien kombiniert. Der neue CT-Prototyp wurde bereits erfolgreich mit einem Thorax Phantom getestet, und ist groß genug für den geplanten Einsatz bei Patientinnen und Patienten.

\section{Dunkelfeld-Röntgen}

Dunkelfeld-Röntgen nutzt die Streuung des Röntgenlichts. Trifft Röntgenlicht auf Materialien unterschiedlicher Dichte, wie zum Beispiel an den Grenzflächen zwischen Lungengewebe und Luft, wird es kleinwinklig gestreut. Wertet man diese Kleinwinkelstreuung aus, erhält man zusätzliche Informationen über feinste Gewebestrukturen, die mit konventionellen Röntgenverfahren nicht auflösbar wären.

Um das gestreute Röntgenlicht detektieren zu können, sind spezielle mikrostrukturierte Gitter nötig. Diese werden zwischen Röntgenquelle und Detektor angebracht. Wenn das Röntgenlicht die Gitter passiert, entsteht ein charakteristisches Muster auf dem Detektor. Platziert man eine Probe oder Person zwischen den Gittern, verändert sich das Muster. Dadurch sind Rückschlüsse auf die Struktur der Probe oder das Gewebe der Person möglich.
Zusätzliche Informationen für Diagnosen in der Klinik

Mit dem Dunkelfeld-CT-Prototyp können beim gleichen Scan-Durchgang konventionelle Röntgenaufnahmen und DunkelfeldAufnahmen gemacht werden. Die dadurch gewonnen zusätzlichen Informationen könnten zukünftig nicht nur bei der Diagnose von Lungenkrankheiten, sondern beispielsweise auch bei der Diagnose von Nierensteinen und Ablagerungen im Gewebe von Vorteil sein.

Die Forschenden planen, die Bildgebung mit dem Dunkelfeld-Computertomographen im nächsten Schritt noch weiter zu optimieren und das Gerät für den ersten Einsatz bei Patientinnen und Patienten vorzubereiten.

\section{Literatur}

Viermetz M, Gustschin N, Schmid C et al (2022) Dark-field computed tomography reaches the human scale. PNAS DOI: https://doi.org/10.1073/pnas.2118799119

Quelle: Technische Universität München 
Hier steht eine Anzeige.

黑 Springer 